\title{
Orientation of the cross-field anisotropy of small-scale ionospheric irregularities and direction of plasma convection
}

\author{
E. D. Tereshchenko ${ }^{1}$, N. Yu. Romanova ${ }^{1}$, and A. V. Koustov ${ }^{2}$ \\ ${ }^{1}$ Polar Geophysical Institute of Russian Academy of Sciences, 15 Khalturina, 183010 Murmansk, Russia \\ ${ }^{2}$ Institute of Space and Atmospheric Studies, University of Saskatchewan, Saskatoon, Canada
}

Received: 15 November 2004 - Revised: 11 March 2005 - Accepted: 22 March 2005 - Published: 3 June 2005

\begin{abstract}
The relationship between the orientation of the small-scale ionospheric irregularity anisotropy in a plane perpendicular to the geomagnetic field and the direction of plasma convection in the $\mathrm{F}$ region is investigated. The crossfield anisotropy of irregularities is obtained by fitting theoretical expectations for the amplitude scintillations of satellite radio signals to the actual measurements. Information on plasma convection was provided by the SuperDARN HF radars. Joint satellite/radar observations in both the auroral zone and the polar cap are considered. It is shown that the irregularity cross-field anisotropy agrees quite well with the direction of plasma convection with the best agreement for events with quasi-stationary convection patterns.
\end{abstract}

Keywords. Ionosphere (Auroral ionosphere; Ionospheric irregularities)

\section{Introduction}

The high-latitude ionosphere is an inhomogeneous media in which the quasi-layered distribution of electron density with height also changes horizontally, with spatial scales from hundreds to tens of kilometers. In addition to the largescale structuring, much finer irregularities of the electron density are often observed, more frequently at the edges of large-scale structures (Tsunoda, 1988). Such irregularities can be of various scales, from kilometers to centimeters; they are often referred to as the small-scale irregularities. It is well established that small-scale irregularities are generated in the high-latitude ionosphere through various plasma instabilities (e.g. Keskinen and Ossakow, 1983; Tsunoda, 1988; Gondarenko and Guzdar, 2004). Both theory and observations indicate that the small-scale irregularities are anisotropic; they are strongly stretched along the geomagnetic field and often have a preferential direction in a plane perpendicular to the geomagnetic field; in this paper, the di-

Correspondence to: N. Yu. Romanova

(romanova@pgi.ru) rection of this elongation will be called the orientation of the cross-field anisotropy.

Various parameters of small-scale ionospheric irregularities can be measured by radio methods (e.g. Gusev and Ovchinnikova, 1980; Ruohoniemi et al., 1987; Afraimovich et al., 2001), and numerous results have been reported in the past (e.g. Moorcroft and Arima, 1972; Martin and Aarons, 1977; Fremouw et al., 1977; Rino et al., 1978; Rino and Livingston, 1982; Gailit et al., 1982; Eglitis et al., 1998). Despite significant progress in this field, the relationship between the irregularity parameters and the conditions in the background ionospheric plasma is not well established.

Recently, Tereshchenko et al. (1999) developed a new method of satellite signals analysis that allows one to infer such important characteristics of the ionospheric anisotropic irregularities as the degree of their stretching along and perpendicular to the geomagnetic field and the orientation of the cross-field anisotropy. Further expansions of this method were recently presented in Tereshchenko et al. (2004). Tereshchenko et al. (2000a) applied the original method to the analysis of auroral zone irregularities and, by comparing the inferred orientations of cross-field anisotropy with the direction of plasma convection, as measured by the EISCAT incoherent scatter radar, found their reasonable agreement. Since the joint satellite-EISCAT data set was limited, Tereshchenko et al. (2002) expanded the investigation of the orientation of the cross-field anisotropy by involving the Heppner and Maynard's plasma convection model (Rich and Maynard, 1989). Again, reasonable agreement was found between the orientation of the cross-field anisotropy and the plasma convection direction given by the model for specified conditions. It was noted that occasionally the inferred orientation of the cross-field anisotropy was quite different at two closely spaced receiver sites $(\sim 100 \mathrm{~km})$. These inconsistencies were attributed to strong spatial variations of plasma flow, though no supporting data were provided.

This study continues the investigation of the relationship between the orientation of the cross-field anisotropy of ionospheric irregularities and the direction of plasma convection. 
We compare Tereshchenko et al. $(1999,2004)$ method predictions with convection data provided by the Super Dual Auroral Radar Network (SuperDARN) HF radars. The advantage of the SuperDARN radars for this kind of work is in their capability to monitor the plasma convection in spatially extensive areas of the high-latitude ionosphere with temporal and spatial resolutions of 1-2 min and $\sim 45 \mathrm{~km}$, respectively. We consider three different experiments. The first two were carried out in the auroral zone, and these comparisons expand the previous analysis by Tereshchenko et al. (2000a; 2002). We then consider the third experiment with observations in the polar cap, where the geophysical conditions for ionospheric irregularity formation can be different.

\section{Determination of the irregularity parameters from amplitude scintillations of satellite signals}

Tereshchenko et al. (1999, 2000a,b, 2004) presented details of their method that allows one to infer several characteristics of the ionospheric irregularities from scintillations of the satellite signal amplitudes measured on the ground. Here we briefly give an overview of the method and demonstrate some of its features. The method is based on the so-called Rytov's approach (Rytov et al., 1978). It is assumed that there is an ionospheric layer homogeneously filled with threedimensional (anisotropic) irregularities of electron density. The irregularity spectrum as a function of wave number is described by the power law with an arbitrary index. For satellite signals passing such a layer, the variance of the logarithm of the signal amplitude relative to the signal amplitude in the irregularity-free situation $\sigma_{\chi}^{2}$ is predicted theoretically and compared with measurements. This parameter was selected for the comparison because it is very sensitive to an assumed shape of the irregularities, including the orientation of the cross-field anisotropy.

\subsection{Basics of the theory}

According to Tereshchenko et al. (2004), the variance of the logarithm of the relative amplitude $\sigma_{\chi}^{2}$ is

$$
\begin{aligned}
& \sigma_{\chi}^{2}=\frac{\lambda^{2} r_{e}^{2} \alpha \beta L_{0}^{3-p} \pi^{(p-1) / 2}}{2^{-p / 2+3} \sin \left(\frac{\pi}{4}(p-2)\right) \Gamma((p-3) / 2)} \int_{z_{l}}^{z_{u}} \frac{\sigma^{2}(z)}{\sqrt{1+\gamma(z)}} R_{F}^{p-2}(z) \\
& \times F\left(1-\frac{p}{2}, \frac{1}{2}, 1, \frac{\gamma(z)}{1+\gamma(z)}\right)\left[c(z)-\sqrt{a^{2}(z)+b^{2}(z)}\right]^{-p / 2} d z,
\end{aligned}
$$

where

$$
\chi=\ln \frac{A}{A_{0}} \text {. }
$$

In Eq. (2), $A$ is the measured signal amplitude, and $A_{0}$ is the signal amplitude that would be measured in the absence of ionospheric irregularities.

In expression (1)

$$
\gamma(z)=\frac{2 \sqrt{a^{2}(z)+b^{2}(z)}}{c(z)-\sqrt{a^{2}(z)+b^{2}(z)}},
$$

$a=\frac{1}{2}\left[\left(\alpha^{2}-1\right) \sin ^{2} \theta(z)+\left(\beta^{2}-1\right)\left(\sin ^{2} \psi \cos ^{2} \theta(z)-\cos ^{2} \psi\right)\right]$,

$b=\left(\beta^{2}-1\right) \sin \psi \cos \psi \cos \theta(z)$,

$c=1+\frac{1}{2}\left[\left(\alpha^{2}-1\right) \sin ^{2} \theta(z)+\left(\beta^{2}-1\right)\left(\sin ^{2} \psi \cos ^{2} \theta(z)+\cos ^{2} \psi\right)\right]$,

where $F\left(1-\frac{p}{2}, \frac{1}{2}, 1, \frac{\gamma(z)}{1+\gamma(z)}\right)$ is the hypergeometric function, $\lambda$ is the wave length of the radio signal, $r_{e}$ is the classical electron radius, $R_{F}$ is the Fresnel radius, $z_{l}$ and $z_{u}$ are the lower and upper boundaries of the irregularity layer, the $z$ axis is assumed to coincide with the look direction from the receiver to the satellite, $\Gamma$ is the gamma-function, $p$ is the power index, $L_{0}$ is the outer scale of irregularities, $\theta(z)$ is the angle between the satellite-receiver direction and the vector of the local geomagnetic field, $\alpha$ and $\beta$ are field-aligned and cross-field elongations of the irregularities, and $\Psi$ is the orientation angle of the cross-field anisotropy (the angle is counted from geographic north, positive clockwise). We should note that the Rytov's approach is valid for weak scintillations, i.e. for those with $\sigma_{\chi}^{2}<0.3$. Numerous measurements of the amplitude fluctuations in the subauroral and auroral ionospheres showed that this condition is met for most of cases (Aarons, 1982).

Tereshchenko et al. (1999) proposed to plot the experimentally determined $\sigma_{\chi}^{2}$ in terms of satellite position along the meridian and then to compare this curve with a set of theoretically expected dependencies. One can then find the best-fit theoretical curve to the measured profiles of $\sigma_{\chi}^{2}$ and thus infer the irregularity parameters $\alpha, \beta$ and $\Psi$. The fitting procedure is greatly simplified by the fact that the latitudinal location of the $\sigma_{\chi}^{2}$ theoretical maximum depends solely on the angle $\Psi$ while the shape of the $\sigma_{\chi}^{2}$ theoretical profile depends on $\alpha$ and $\beta$. Note that the maximum amplitude scintillations occur for satellite positions in the vicinity of the magnetic zenith. The success of the fitting procedure depends on whether the satellite pass is near the magnetic zenith or away from it. To characterize how far the satellite path was from the magnetic zenith, the minimum look angle $\theta_{\min }$ (from a receiver to a satellite) and the local direction of the geomagnetic field is considered. For the case of the magnetic-zenith path $\left(\theta_{\min }<1^{\circ}\right)$, the maximum of scintillations occurs exactly at the magnetic zenith, and only parameter $\alpha$ can be determined since signal oscillations originate from isotropic irregularities with $\beta=1$. For a non-zenith path, the shape of the $\sigma_{\chi}^{2}$ profile is also influenced by anisotropic irregularities $(\beta>1)$, so that both $\alpha$ and $\beta$ can be determined. Note that in this case, the $\sigma_{\chi}^{2}$ maximum does not exactly correspond to the satellite position with $\theta=\theta_{\min }$.

\subsection{Measurements of $\Psi$ : zenith and non-zenith satellite} passes

Figure 1 shows experimental (solid line) and theoretical (crosses and dots) curves for the variance of the logarithm of the relative amplitude $\sigma_{\chi}^{2}$ versus geographic latitude. Both cases of a) near zenith and $b$ ) non-zenith satellite passes are considered, with angles $\theta_{\min }$ of $0.5^{\circ}$ and $7.3^{\circ}$, respectively. 
The scintillation data were collected at a receiver site located on the Kola Peninsula. For modeling, it was assumed that the ionospheric irregularities filled a statistically homogeneous layer with boundaries $z_{l}=250 \mathrm{~km}$ and $z_{u}=350 \mathrm{~km}$ and that the variance of the electron density fluctuations was the same at all heights. Theoretical predictions are shown for two values of $\Psi$, in each case a) and b); one value corresponds to the case of the best fit between the theory and measurements (dots) and the other one (crosses) corresponds to the case of a significantly different angle $\Psi$; we selected this angle to be $40^{\circ}$ away (anticlockwise) from the direction of the best fit. This second value of $\Psi$ is considered to demonstrate how sensitive the position of the theoretical maximum to the choice of $\Psi$ is, for both zenith and non-zenith satellite passes.

In case a), the best fit is achieved for $\alpha=55, \beta=1$ and $\Psi=106^{\circ}$. In case b), the best fit is obtained for $\alpha=30, \beta=5$ and $\Psi=79^{\circ}$. The $40^{\circ}$ offset in the angle $\Psi$ changes significantly (not significantly) the position of the $\sigma_{\chi}^{2}$ theoretical maximum for the non-zenith (zenith) pass. This implies that the angle $\Psi$ can be inferred quite accurately from the experimental data for non-zenith satellite passes. We performed extensive analysis of the satellite data and found that, for nonzenith passes, a $4^{\circ}-6^{\circ}$ variation in $\Psi$ changes noticeably the position of the theoretical maximum for $\sigma_{\chi}^{2}$. We also found that the horizontally anisotropic irregularities become detectable starting from $\theta_{\min }=1^{\circ}-1.5^{\circ}$. Luckily, for most satellite trajectories the magnitude of $\theta_{\min }$ exceeds these minimum values. We estimated the uncertainty in the determination of $\Psi$ by finding a set of $\Psi$ values for which the difference between the experimental and theoretical $\sigma_{\chi}^{2}$ curves was not significant. For $\theta_{\min }>1.5^{\circ}$, the uncertainty is of the order of $2^{\circ}-6^{\circ}$ and it increases for $\theta_{\min }<1.5^{\circ}$. We should note that the uncertainty in the determination of $\alpha$ and $\beta$ is larger; it ranges from several units of elongation $(10 \%-20 \%$ effect) to a difference $(100 \%-300 \%$ effect) of two or more. What is important though is the fact that the larger uncertainty in the determination of $\alpha$ and $\beta$ does not affect the uncertainty in the determination of $\Psi$.

The analysis performed allowed us to conclude that the value of $\Psi$ can be determined very reliably from the amplitude scintillations of the satellite signals.

\subsection{Determination of the parameters $\alpha$ and $\beta$}

Now we demonstrate how the magnetic field elongation of ionospheric irregularities (parameter $\alpha$ ) and cross-field anisotropy (parameter $\beta$ ) can be determined from satellite scintillation data. Figure 2 shows the theoretical profiles for $\sigma_{\chi}^{2}$ versus geographical latitude for two values of $\beta$, dots for the optimal value of $\beta>1$ and crosses for the case of isotropic irregularities $(\beta=1)$. The experimental data were obtained on 16 November 1997, 21:34 UT at three receiver sites in Norway: Kårvika $\left(69.87^{\circ} \mathrm{N}, 18.93^{\circ} \mathrm{E}\right)$, Troms $\varnothing\left(69.59^{\circ} \mathrm{N}\right.$, $\left.19.22^{\circ} \mathrm{E}\right)$ and Nordkjosbotn $\left(69.22^{\circ} \mathrm{N}, 19.54^{\circ} \mathrm{E}\right)$. The satellite trajectories in all three cases were of non-zenith type; the minimum angles between the line of sight to the satellite and the geomagnetic field were $\theta_{\min }=7.2^{\circ}$ in Kårvika, $\theta_{\min }=7.2^{\circ}$
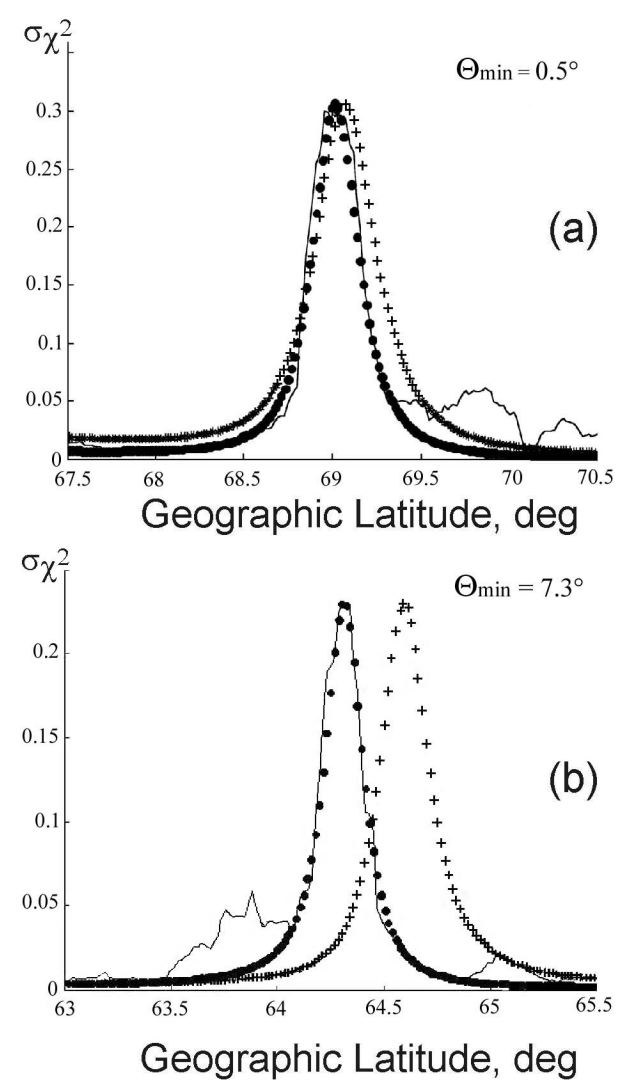

Fig. 1. Experimental (solid line) and theoretical (dots and crosses) latitudinal profiles (the geographic latitude is used) of the logarithm of the relative amplitude of a satellite signal $\left(\sigma_{\chi}^{2}\right)$ for (a) near zenith and (b) non-zenith passes over a receiver site on the Kola Peninsula. Dots correspond to the case of the best fit between the model and experiment and crosses correspond to the case of the cross-field anisotropy orientation rotated by $40^{\circ}$ anticlockwise from the direction of the best fit. Also shown is the minimum angle $\theta_{\text {min }}$ between the look direction from the receiver site to the satellite and the local geomagnetic field direction at the $\mathrm{F}$ region heights.

in Troms $\varnothing$ and $\theta_{\min }=7.4^{\circ}$ in Nordkjosbotn. We indicate on each panel the irregularity parameters for the case of the best fit between the experimental and theoretical curves. One can see that the position of the theoretical maximum is not very sensitive to the choice of $\beta$ in all three cases (whether it is 1 or 7). On the other hand, the width of the curves is strongly affected by $\beta$. Detailed analysis shows that variations of the parameter $\alpha$ change the shape of the $\sigma_{\chi}^{2}$ curve near the maximum while variations of the parameter $\beta$ strongly control the "tails" of the $\sigma_{\chi}^{2}$ curve; generally, a decrease (increase) of either $\alpha$ or $\beta$ makes the $\sigma_{\chi}^{2}$ profiles broader (narrower).

Importantly, the analysis shows that a uniquely defined set of $\alpha$ and $\beta$ can be found for each satellite pass, if the best fit between the theoretical and experimental profiles of $\sigma_{\chi}^{2}$ is sought. One can also conclude from Fig. 2 that the experimental curves cannot be described by the model of isotropic irregularities for non-zenith passes; this is in contrast to the case of the almost-zenith pass considered in Fig. 1a. 
16 November 1997, 21:34 UT
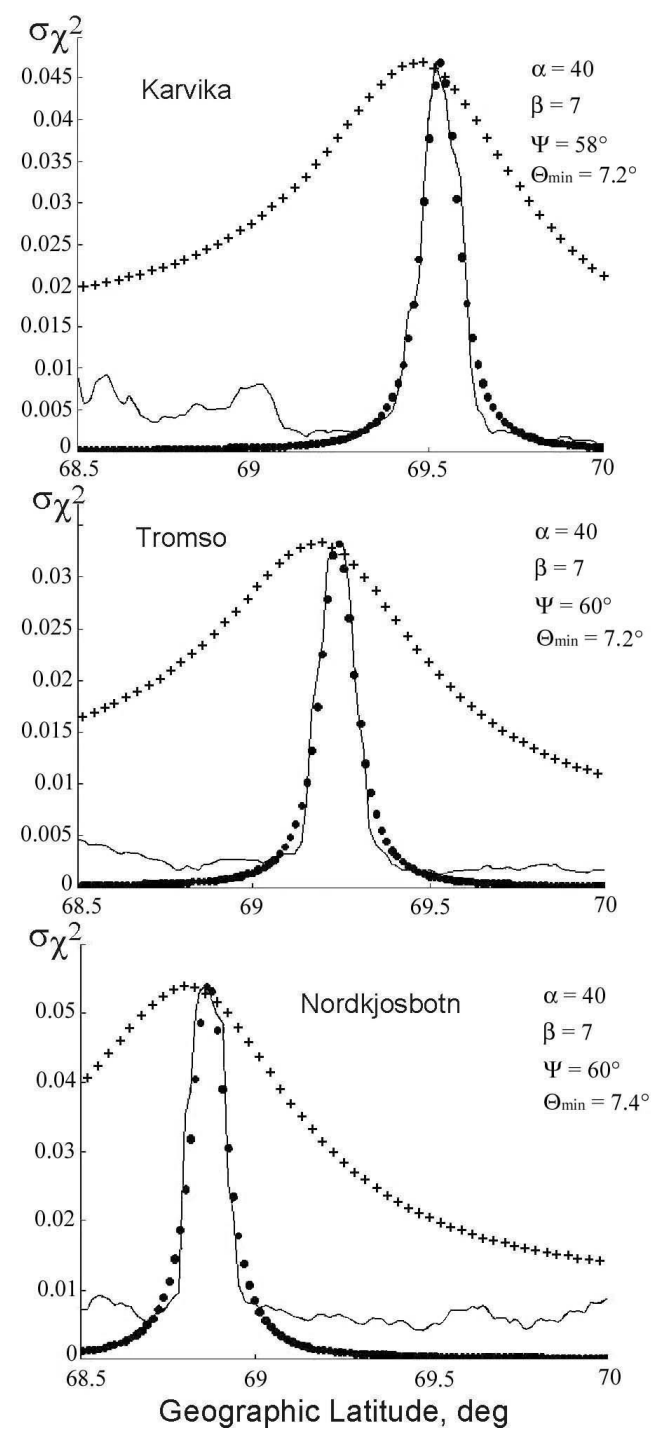

Fig. 2. Experimental (solid line) and theoretical (dots and crosses) latitudinal profiles of the logarithm of the relative amplitude of a satellite signal $\left(\sigma_{\chi}^{2}\right)$ for a pass over Kårvika, Troms $\emptyset$ and Nordkjosbotn (all in northern Norway) on 16 November 1997 at $~ 21: 34$ UT. Geographic latitudes are used. Dots (crosses) correspond to the case of anisotropic $\beta>1$ (isotropic, $\beta=1$ ) ionospheric irregularities. The irregularity parameters for the best fit between the experimental and model profiles are given in the upper right corner of each diagram.

2.4 Multi-receiver observations: some conclusions on the ionospheric conditions

The Tereshchenko et al. (1999) method allows one to infer the irregularity parameters in the ionospheric region above the receiver location. If data of several receivers are compared, conclusions on the spatial homogeneity of the irregularity layer can be drawn.

Consider observations presented in Fig. 2. The estimated irregularity parameters are $\alpha=40, \beta=7, \Psi=58^{\circ}\left( \pm 2^{\circ}\right)$ in Kårvika, $\alpha=40, \beta=7, \Psi=60^{\circ}\left( \pm 2^{\circ}\right)$ in Troms $\varnothing$ and $\alpha=40$, $\beta=7, \Psi=60^{\circ}\left( \pm 2^{\circ}\right)$ in Nordkjosbotn. For all three sites, the data show only one maximum well described by the same value of $\alpha$ and the same value of $\beta$. This implies that the electron density fluctuations (anisotropic irregularities) are of the same character (shape) above these sites, and their distribution is quite uniform. Certainly, this is a very special situation; generally, one cannot expect such a spatial uniformity of the density fluctuations over distances of tens to hundreds of kilometers in the high-latitude ionosphere. In the case of non-uniform irregularity spatial distribution, one can observe more than one peak in the latitudinal profiles of $\sigma_{\chi}^{2}$. Also, for the case of a single maximum in the profile, different values of $\alpha$ and $\beta$ can be obtained even at close receiver locations.

Figure 3 presents experimental $\sigma_{\chi}^{2}$ curves obtained at Kårvika, Troms $\varnothing$ and Nordkjosbotn on 14 November 1997 at 18:28 UT and corresponding theoretical profiles. The best parameters describing the data are $\alpha=20, \beta=4, \Psi=91^{\circ}\left( \pm 3^{\circ}\right)$ for Kårvika, $\alpha=20, \beta=4, \Psi=120^{\circ}\left( \pm 3^{\circ}\right)$ for Troms $\emptyset$ and $\alpha=25, \beta=5, \Psi=125^{\circ}\left( \pm 3^{\circ}\right)$ for Nordkjosbotn. In this case, only Troms $\varnothing$ and Kårvika data can be described by the same model of irregularities, though the orientation of the crossfield anisotropy is different at these locations. Different orientation of the cross-field anisotropy over Kårvika occurred, very likely, because of a change in the direction of plasma convection, as suggested for similar cases by Tereshchenko et al. (2002).

We should note that some observations do not show a clear maximum for the $\sigma_{\chi}^{2}$ profile, so that the irregularity parameters cannot be determined at all. One example is given in Figure 4 for 9 November 1997 at 15:10 UT. Here the welldefined isolated maxima are seen at Kårvika and Troms $\varnothing$; best fitting for these data gives $\alpha=30, \beta=6, \Psi=41^{\circ}\left( \pm 3^{\circ}\right)$ at Kårvika and $\alpha=30, \beta=6, \Psi=60^{\circ}\left( \pm 3^{\circ}\right)$ at Troms $\varnothing$. We cannot determine the irregularity parameters over Nordkjosbotn. The most likely reason is that the irregularities were very weak or patchy. During the period of 15:08-15:18 UT, the Troms $\emptyset$ heater was producing artificial irregularities near the zenith of the station. This allowed us to reliably determine the irregularity parameters at this location. Since plasma convection was directed poleward, the artificially generated irregularities were drifting poleward and strong satellite signals scintillations were seen at Kårvika. The artificial irregularities were not able to reach Nordkjosbotn while the background fluctuations were probably too weak to produce strong scintillations. Similar situations were described by Tereshchenko et al. (2000a,b); we present here the additional case to illustrate and stress some limitations of the method.

The data presented in this section demonstrate that a network of satellite signal receivers separated by less than one hundred kilometers can provide important information on the fine structure of the high-latitude ionosphere.

\subsection{On the role of time-averaging in the model}

The approach based on Eq. (1), that we have discussed so far, has a minor inconsistency in terms of data handling and modelling. When experimental data are processed, the $\sigma_{\chi}^{2}$ 


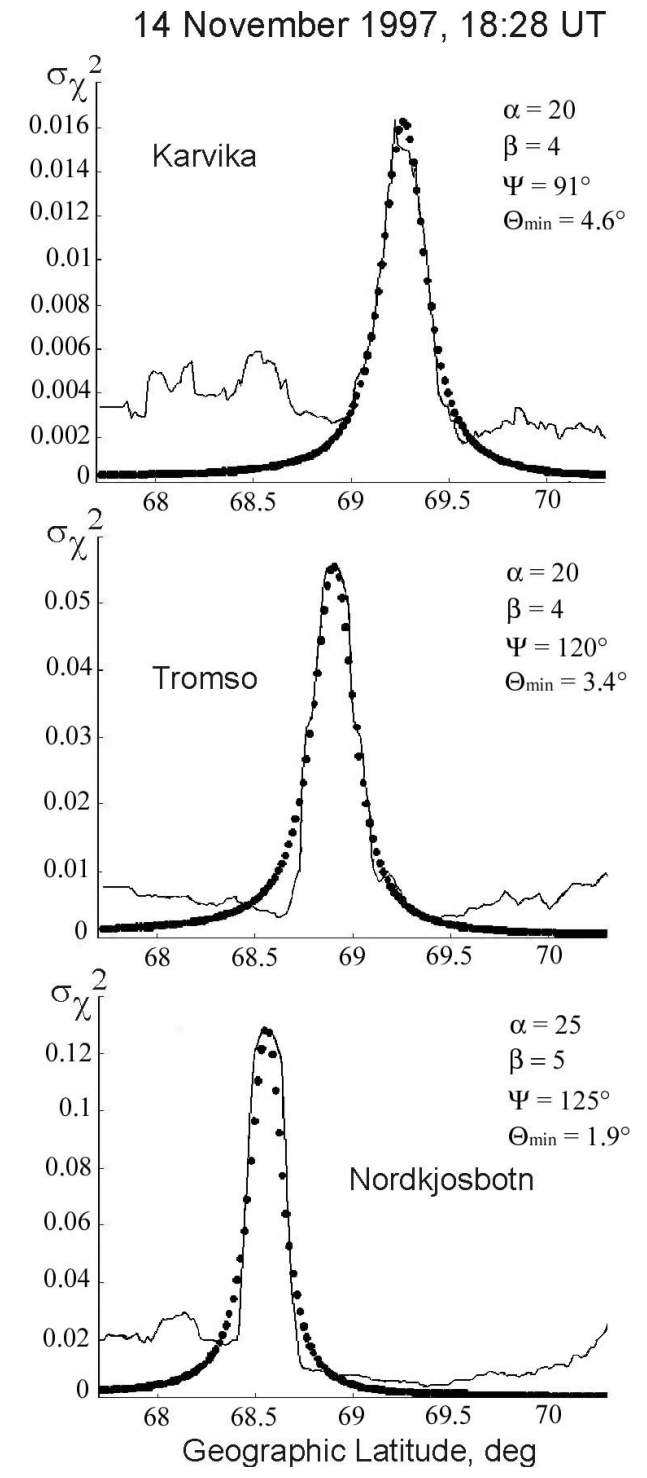

Fig. 3. The same as in Fig. 2 but for 14 November 1997, 18:28 UT.

profile is obtained by computing $\sigma_{\chi}^{2}$ for every 8 -12-s period and merging all obtained values into one latitudinal profile. The theoretical curve is obtained by computing $\sigma_{\chi}^{2}$ at every instant of time, for example, for every second (below we will call such a curve/profile "the instantaneous curve/profile"). Clearly, it is desirable to produce the theoretical curve in the same fashion as the experimental one, i.e. instead of an instantaneous value of $\sigma_{\chi}^{2}$ for every second, we consider the $\sigma_{\chi}^{2}$ value averaged over $8-12 \mathrm{~s}$. In this section we investigate the significance of this averaging effect and its potential impact on the determination of parameters $\alpha$ and $\beta$. Our analysis showed that the time averaging does not affect the determination of $\Psi$.

Figure 5a shows averaged (crosses) and instantaneous (solid line) theoretical curves for a near zenith satellite pass over a receiver at Troms $\emptyset$. For the purpose of illustration, we selected typical values of $\alpha=40$ and $\beta=6$. The value of
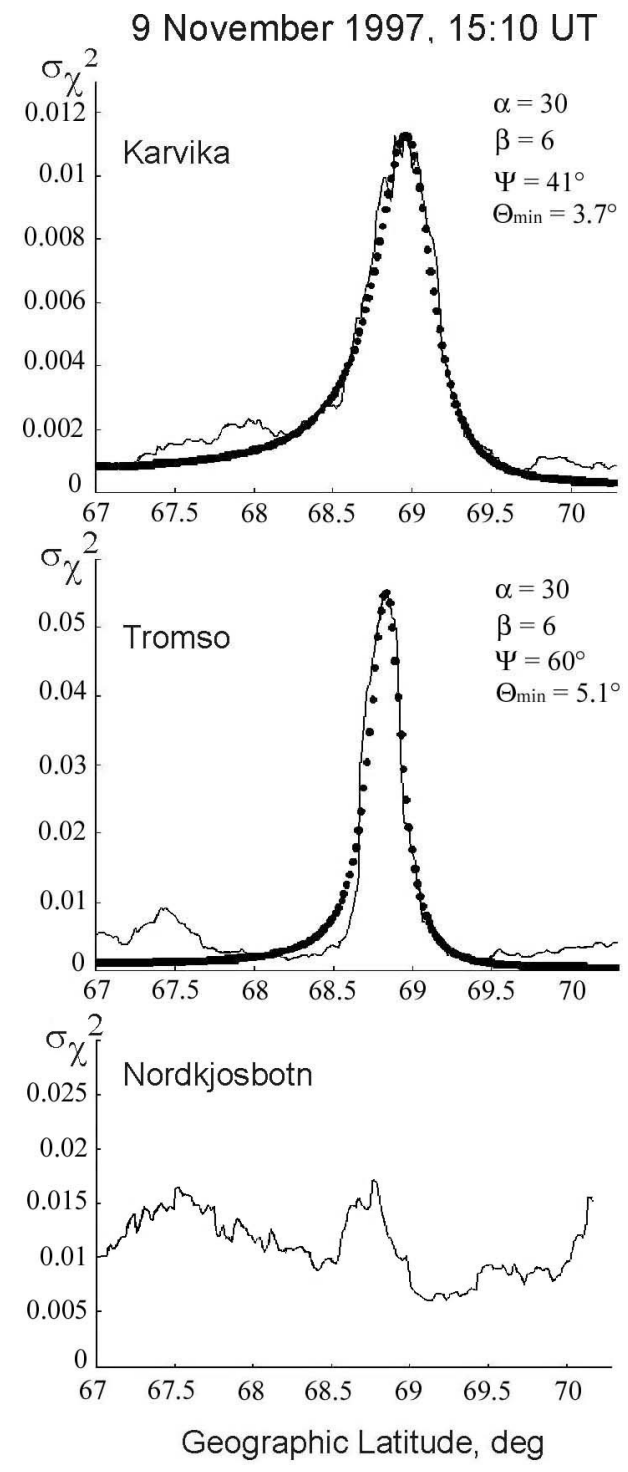

Fig. 4. The same as in Fig. 2 but for 9 November 1997, 15:10 UT. At Nordkjosbotn, the maximum of $\sigma_{\chi}^{2}$ at $\sim 68.7^{\circ}$ was not strong, and the irregularity parameters were not determined.

$\Psi=71^{\circ}$ was selected so that the maximum of the $\sigma_{\chi}^{2}$ instantaneous profile (solid line) is achieved exactly at the angle $\theta_{\min }$. We show the latitudinal variation for $\theta$ by the dotted line in Figure 5a, and one can see that its minimum coincides with the maximum of the $\sigma_{\chi}^{2}$ curve. The model values of the anisotropy parameters are also shown in the figure. The value $\theta_{p}=7.7^{\circ}$ indicates the zenith angle of the satellite position corresponding to the peak in $\sigma_{\chi}^{2}$. One can see in Fig. 5a that the averaged profile of $\sigma_{\chi}^{2}$ (crosses) reaches its maximum at $\theta=\theta_{\min }$ (at the same latitude as for the instantaneous profile), but its magnitude is smaller than the maximum of the instantaneous profile. Varying the parameter $\Psi$ only shifts both the instantaneous and averaged curves horizontally (without the curves' distortion), indicating that the averaging effect is controlled by only $\alpha$ and $\beta$. We found that the case of Fig. 5a is a very typical situation for many passes. 

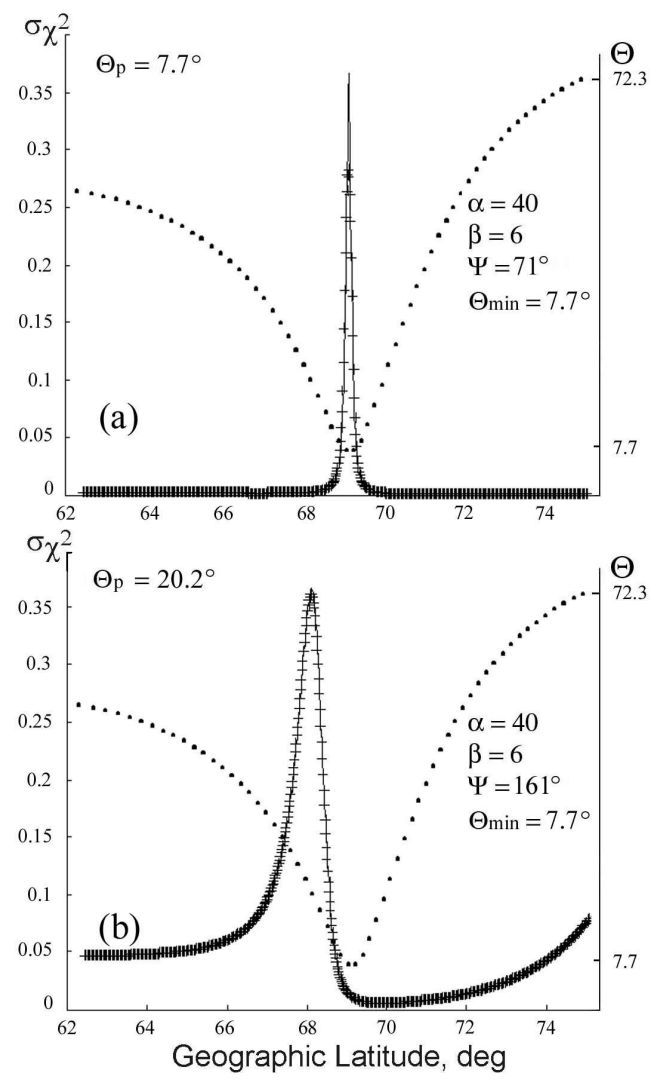

Fig. 5. Averaged (crosses) and instantaneous (solid line) theoretical variations of $\sigma_{\chi}^{2}$ versus geographic latitude for a receiver at Troms $\emptyset$. Panel a) corresponds to the case of the $\sigma_{\chi}^{2}$ maximum (achieved at the angle $\theta=\theta_{p}=7.7^{\circ}$ ) which is exactly at the latitude of $\theta_{\min }$ while panel b) corresponds to the case of the $\sigma_{\chi}^{2}$ maximum (achieved at the angle $\theta=\theta_{p}=20.2^{\circ}$ ) located at the latitude lower than the latitude of $\theta_{\min }$. Computations were performed for the integration time of $10 \mathrm{~s}$ and typical irregularity parameters were $\alpha=40$ and $\beta=6$. Dots show the latitudinal variation of the angle $\theta$.

For some passes and irregularity parameters the difference between the averaged and instantaneous curves is not significant. Figure $5 \mathrm{~b}$ illustrates such a situation for observations over Troms $\varnothing$. Here we consider the pass with $\theta_{\min }=7.7^{\circ}$ and the same parameters $\alpha$ and $\beta$ as in the previous case, but the orientation of the cross-field anisotropy (parameter $\Psi_{A}$ ) is different. One can see that the instantaneous (solid line) and averaged (crosses) $\sigma_{\chi}^{2}$ curves coincide and both maxima are achieved at the look angle of $\theta_{p}=20.2^{\circ}$, i.e. significantly away from the magnetic zenith.

By considering various satellite passes and varying the irregularity parameters we were able to draw three general conclusions. First, if an instantaneous $\sigma_{\chi}^{2}$ curve has its maximum near the point $\theta=\theta_{\min }$, then the averaging effect is not significant for small $\alpha$ and when $\beta<\alpha$. For example, if averaging is done over $10 \mathrm{~s}, \alpha$ should be less than $10-12$. This implies that the irregularities should be moderately anisotropic to neglect the averaging in the model. Second, for strongly anisotropic irregularities (for example, $\alpha$ more than 10 and $\beta<\alpha$ ), the averaging effect is not significant for satellite passes with the $\sigma_{\chi}^{2}$ maximum achieved at large angles $\theta$ of $\sim 15^{\circ}-20^{\circ}$. Third, the averaging effect is less significant if an instantaneous curve has its maximum away from the point $\theta=\theta_{\min }$. Finally, we found that consideration of the averaging effect is more important for the determination of $\alpha$; values of $\beta$ usually do not change significantly.

To give a sense of the error in estimation of $\alpha$ and $\beta$ we consider the case of Figure 5a. Application of our standard procedure (without considering the averaging effect) to the averaged curve (crosses) gives $\alpha=31$ and $\beta=6$. We see that $\beta$ did not change while $\alpha$ is now smaller by 9 (31 versus 40). This means that if the instantaneous theoretical curve (solid line) is fit to the experimental curve for the considered pass (so that the averaging effect is ignored), then the derived value of $\alpha$ is smaller than it should be.

\section{Results of joint SuperDARN - satellite signal observa- tions}

In this study we consider data collected in three independent experiments. The first experiment was run between 9 and 15 November 1997 in northern Norway, in conjunction with ionospheric HF heating (Tereshchenko et al., 2000b). The satellite signal reception was conducted at three sites, Kårvika $\left(69.87^{\circ} \mathrm{N}, 18.93^{\circ} \mathrm{E}\right)$, Troms $\varnothing\left(69.59^{\circ} \mathrm{N}\right.$, $\left.19.22^{\circ} \mathrm{E}\right)$ and Nordkjosbotn $\left(69.22^{\circ} \mathrm{N}, 19.54^{\circ} \mathrm{E}\right)$, separated by $\sim 100 \mathrm{~km}$. The SuperDARN radars were operated in the standard mode with 2-min scanning through the field of view. Thirteen events of joint radar-satellite data were identified and studied.

The second experiment of a similar type was run in June 2001, with the exception that the satellite signal reception was performed at Futrikelv $\left(69.80^{\circ} \mathrm{N}, 19.02^{\circ} \mathrm{E}\right)$, Troms $\varnothing$ $\left(69.59^{\circ} \mathrm{N}, 19.22^{\circ} \mathrm{E}\right)$ and Seljelvnes $\left(69.25^{\circ} \mathrm{N}, 19.43^{\circ} \mathrm{E}\right)$, also separated by $\sim 100 \mathrm{~km}$. Seven events were considered for this experiment.

The third experiment was conducted on Spitsbergen archipelago, at a settlement of Barentsburg $\left(78.1^{\circ} \mathrm{N}\right.$, $14.21^{\circ} \mathrm{E}$ ) from September 2000 to April 2001. We obtained 28 joint events for this experiment.

For the readers convenience, we remind one that SuperDARN is a network of HF radars continuously monitoring echoes from the high-latitude ionosphere (Greenwald et al., 1995). Currently, SuperDARN consists of 9 radars in the Northern Hemisphere and 7 radars in the Southern Hemisphere. It is assumed that the Doppler frequency of the echoes is the line-of-sight (cosine) component of the plasma convection vector. This assumption is justified by the fact that the phase velocity of the $\mathrm{F}$ region decametre irregularities is very close to the drift of the bulk of the plasma (Ruohoniemi et al., 1987). To obtain a map of plasma convection vectors, all available velocity measurements are fit into the convection model, and the optimal solution is found by the least-squares fit procedure (Ruohoniemi and Baker, 1998). Utilization of SuperDARN data is very convenient for the 

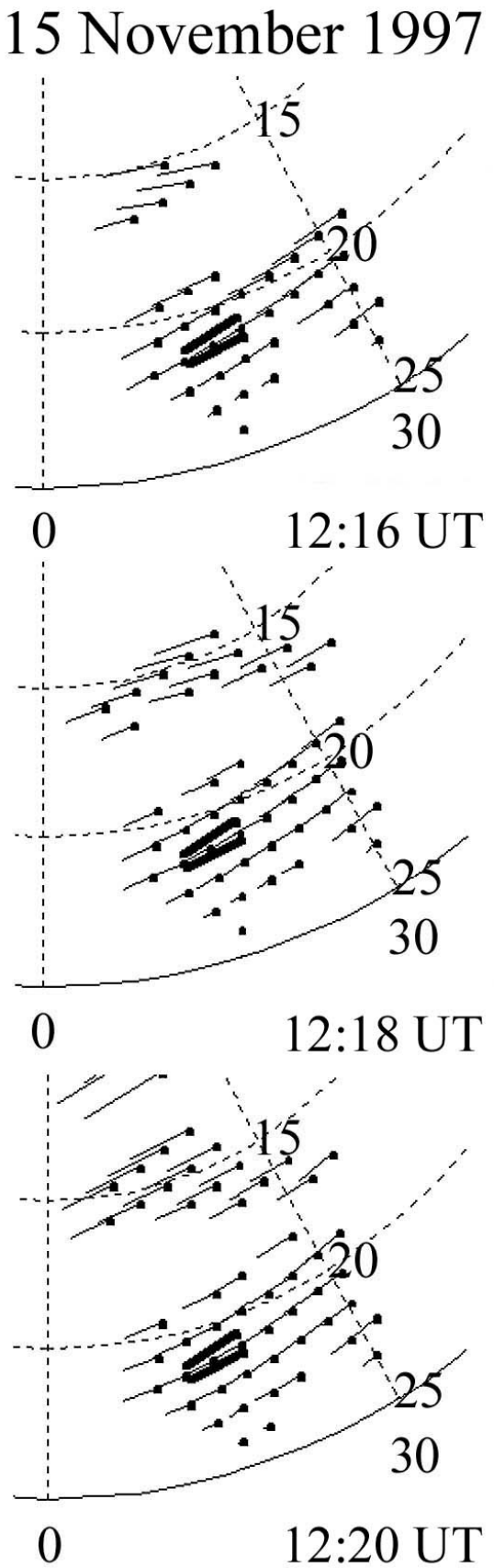

Fig. 6. SuperDARN convection map (thin lines originated at dots) for 15 November 1997 between 12:16 and 12:20 UT and the orientation of the cross-field anisotropy according to measurements at Kårvika and Nordkjosbotn (thick lines) at $\sim 12: 18$ UT. The coordinates are geographic longitude and geographic co-latitude.

purposes of the present work because of the good temporal $(\sim 1-2 \mathrm{~min})$ and spatial $(45 \mathrm{~km})$ resolution of the measurements. In this study, we considered data gathered by all $\mathrm{Su}-$ perDARN radars in the Northern Hemisphere but the major contribution was always made by the Pykkvibaer (Iceland) and Hankasalmi (Finland) radars observing directly in the area of scintillation measurements.
3.1 Auroral zone observations: the case of stationary convection

We first consider results for the auroral zone observations in November 1997. Joint satellite-SuperDARN data were available for various periods in between 12:26 and 22:56 UT (roughly 10:30-21:00 MLT). The orientation of the crossfield anisotropy varied in between $62^{\circ}$ and $125^{\circ}$, clustering at $75^{\circ}-90^{\circ}$. We remind one that the angles are counted from geographic north, clockwise. We should note that the orientation of the cross-field anisotropy can only be determined up to the constant of $180^{\circ}$. We conclude that the overall orientation of the cross-field anisotropy is consistent with the prevailing direction of the plasma convection at the latitudes of the auroral oval.

Let us now show some individual measurements. In 8 cases out of 13 events, the convection and satellite data were for the same area and the satellite-radar data were compared quantitatively. Figure 6 gives an example of such a comparison for 15 November 1997. Here the SuperDARN convection maps (thin vectors originating from the dots) are given for 12:16, 12:18 and 12:20 UT, together with the orientation of the cross-field anisotropy of ionospheric irregularities at Kårvika and Nordkjosbotn (thick vectors). The data on this map (and all others, considered in this study) are presented in geographic coordinates. In the past, Tereshchenko et al. (2000a) used geomagnetic coordinates. This difference is not important for this study, as the target of the investigation is the azimuthal difference between the irregularity elongation and the convection direction, which is independent of the coordinate system used.

At Troms $\varnothing$ there was no strong maximum in the $\sigma_{\chi}^{2}$ curve, and these measurements are not considered. The scintillation measurements refer to 12:18 UT. The orientation of the cross-field anisotropy was $\Psi_{K}=257^{\circ}\left( \pm 3^{\circ}\right)$ in Kårvika and $\Psi_{N}=262^{\circ}\left( \pm 3^{\circ}\right)$ in Nordkjosbotn. The convection direction obtained at the nearest SuperDARN point at 12:18 UT was $\Psi_{S D}=267^{\circ}$. The difference between the orientation of the cross-field anisotropy and the convection direction is $\Delta \Psi_{K}=-10^{\circ}$ for Kårvika and $\Delta \Psi_{N}=-5^{\circ}$ for Nordkjosbotn. Importantly, these differences $\Delta \psi$ are small. This signifies that the small-scale ionospheric irregularities were elongated in the direction of the plasma convection. The fact that the irregularity anisotropy orientations were the same implies that the plasma flow was spatially uniform and this conclusion is consistent with more coarse SuperDARN measurements.

A good agreement between the orientation of the crossfield anisotropy and the convection direction was observed in other cases; we present statistics in Fig. 7 for all 8 events. The data were binned with a $5^{\circ}$-step in the orientation angle. The positive (negative) values denote those measurements for which the satellite-inferred value of $\Psi$ was larger (smaller) than $\Psi_{S D}$. The histogram shows that the differences are less than $5^{\circ}$ in most cases.

In 5 cases for the November 1997 experiment, the information on the convection was not available for the immediate vicinity of scintillation measurements because the 


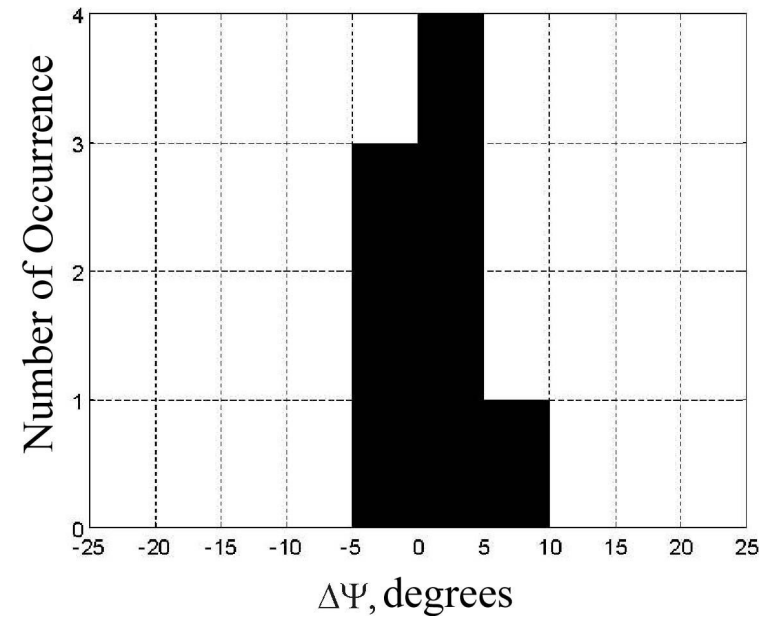

Fig. 7. Histogram distribution for the difference $\Delta \Psi$ between the orientation of the cross-field anisotropy and the direction of the plasma convection for observations between 9 November and 15 November of $1997.5^{\circ}$ bins of the azimuth are used.

SuperDARN data were patchy. For these cases we compared the data only qualitatively. Figure 8 gives an example of such a comparison for 14 November 1997. The scintillation measurements were performed at Troms $\varnothing$ at 17:02 UT. SuperDARN was continuously providing data over 10-min interval of 16:54-17:04 UT, but there were no convection vectors in the region of the scintillation measurements. One can see that the convection pattern is fairly stable, with similar convection vectors to the south, west and east of Troms $\varnothing$. If more vectors were available, we would expect a good agreement between the SuperDARN and scintillation measurements at 17:02 UT (note, that observations such as shown in Fig. 8 were not included in the statistics of Fig. 7). We found a general agreement between satellite and radar measurements for all five events.

\subsection{Auroral zone observations: a case of non-uniform con-} vection

For the second auroral zone experiment, conducted in June 2001, reasonable quality SuperDARN convection maps were obtained for 2, 5 and 11 June. The orientations of the crossfield anisotropy were available for 2 June in Futrikelv and Seljelvnes, for 5 June in Futrikelv, Troms $\varnothing$ and Seljelvnes, and for 11 June in Troms $\emptyset$ and Seljelvnes. Unfortunately, for most of these events, the SuperDARN convection vectors were quite separated from the areas of satellite measurements and we were not able to compare the data quantitatively. Qualitatively, the orientation of the cross-field anisotropy was always in reasonable agreement with the convection direction in nearby regions. Interestingly, for 2 June 2001, the orientation of the cross-field anisotropy and the plasma convection were both in the geographically meridional direction.

Interesting results were obtained for 5 June 2001. In Fig. 9 we show the convection map, together with the satellite measurements of the orientation of the cross-field anisotropy

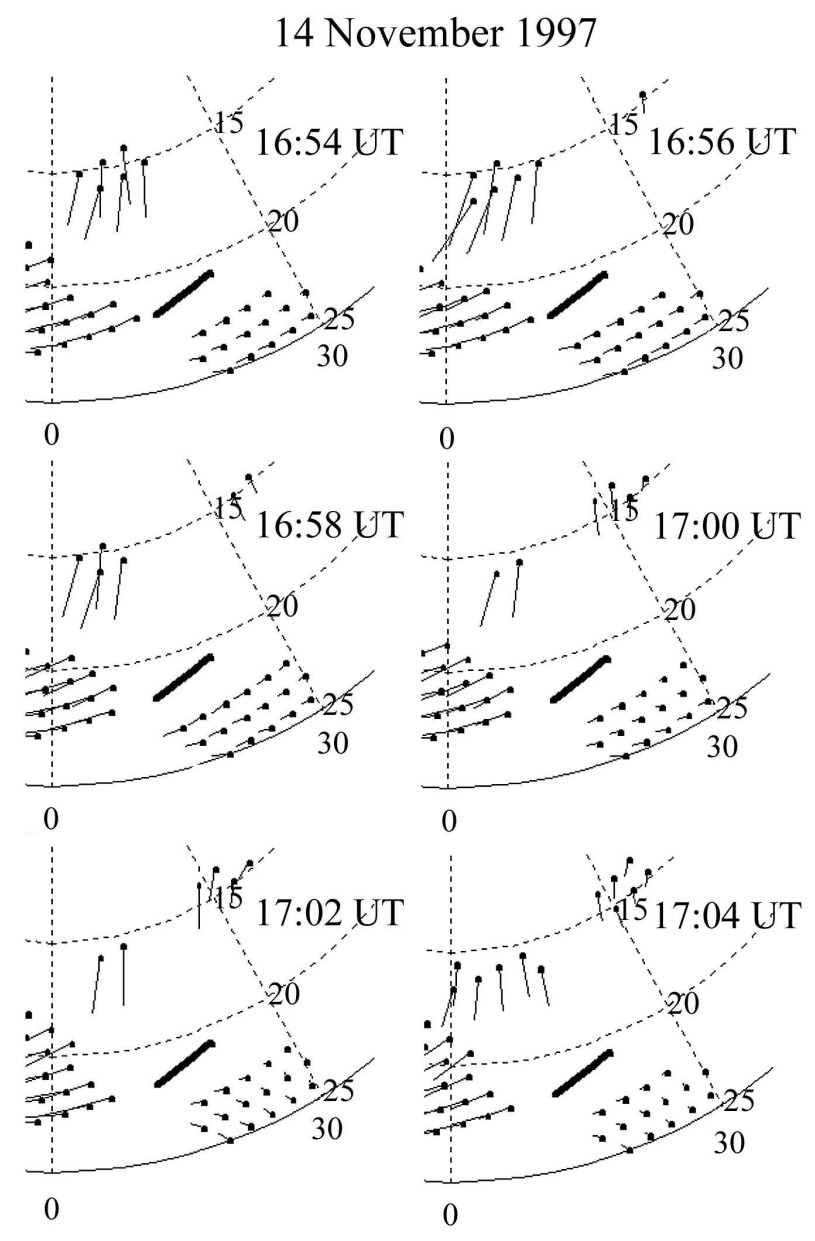

Fig. 8. SuperDARN convection maps (thin lines originating from dots) for 14 November 1997 between 16:54 and 17:04 UT and the orientation of the cross-field anisotropy (thick lines) according to Troms $\varnothing$ measurements at 17:02 UT. The coordinates are geographic longitude and geographic co-latitude.

for 21:08 UT. Convection maps prior to this moment were very similar to the one shown in Fig. 9. For this event, $\Psi_{F}=222^{\circ}\left( \pm 1^{\circ}\right)$ at Futrikelv, $\Psi_{T}=236^{\circ}\left( \pm 2^{\circ}\right)$ at Troms $\varnothing$ and $\Psi_{S}=256^{\circ}\left( \pm 2^{\circ}\right)$ at Seljelvnes. The convection direction measured by SuperDARN at the nearest point was $\Psi_{S D}=256^{\circ}$ so that the differences between the orientation of the cross-field anisotropy and the convection direction were $\Delta \Psi_{F}=-34^{\circ}$ at Futrikelv, $\Delta \Psi_{T}=-20^{\circ}$ at Troms $\varnothing$ and $\Delta \Psi_{S}=0^{\circ}$ at Seljelvnes. Clearly, only the Seljelvnes measurements at the most equatorward site were in good agreement with the convection direction. However, the SuperDARN measurements show that the convection pattern was strongly non-homogeneous; the convection was turning from the eastward flow at the very high latitudes of $\sim 75^{\circ}$ to westward flow at latitudes of $\sim 70^{\circ}$. In other words, the azimuth of the convection vectors was increasing with a decrease of latitude. Thus, there was a correlation in spatial variations of $\Psi$ and $\Psi_{S D}$. The large differences between $\Psi$ and $\Psi_{S D}$ at Futrikelv and Troms $\varnothing$ signify that the convection direction experienced significant 
local variations that were not detected by SuperDARN. This example illustrates the fact that multipoint scintillation observations can be convenient for investigations of local structuring in the plasma flow.

We should say that measurements with $\Psi-\Psi_{S D}$ differences as large as $20^{\circ}$ were not rare for spatially non-uniform or temporally changing convection patterns. For the cases of quasi-stationary convection in time but non-homogeneous in space (around an area of measurements), the angle $\Psi$ was varying with latitude, in general agreement with expected changes of the convection pattern.

Our conclusion from the analysis performed is that multireceiver scintillation measurements can provide additional information on plasma convection and supplement the SuperDARN maps.

\subsection{Polar cap observations}

Now we consider observations on the Spitsbergen archipelago, at the settlement of Barentsburg. The analysis of scintillation data for this location showed that the Tereshchenko et al. (1999) method works quite well for polar cap conditions; the latitudinal profiles of $\sigma_{\chi}^{2}$ show typically a single maximum with values below 0.3 (the criterion for the amplitude scintillations to be treated as small-amplitude ones) and the shape and latitudinal location of the $\sigma_{\chi}^{2}$ curve can well be described by a theoretical curve defined by parameters $\alpha, \beta$ and $\Psi$.

For the Baretsburg observations, measurements covered the time sector of 01:06-23:32 UT (almost all MLT times). The orientation of the cross-field anisotropy varied from $8^{\circ}$ to $173^{\circ}$ with no preferential direction. The reason for this is that the observations were quite frequently carried out near the foci of the large-scale convection cells with rather circular flows, contrary to the zonal flows typical for the mainland Norway observations in the auroral zone.

We split available experimental data into two groups. For the first group, the SuperDARN convection maps were fairly stationary in time. For the second group, the maps showed significant temporal variations. A stationary convection map to us was one for which the convection pattern in the vicinity of scintillation measurements did not show significant changes within several minutes (6-12 min) prior to a moment of the comparison.

Figure 10 gives an example of the SuperDARN/satellite comparison for a relatively stable convection pattern on 5 February 2001, 13:06-13:12 UT. According to scintillation measurements at 13:10 UT, the orientation of the cross-field anisotropy was $\Psi=258^{\circ}\left( \pm 1^{\circ}\right)$. The SuperDARN convection direction at the closest point in time (13:10 UT) and in space was $\Psi_{S D}=259^{\circ}$ so that the difference in angles was small $\Delta \Psi=1^{\circ}$, within the error of measurements. One can see that over a 6-min interval, the convection pattern did not change much in the area of the comparison. The convection directions were $\Psi_{S D}=259^{\circ}-262^{\circ}$. It is not a surprise to see small differences between the convection direction and the orientation of the cross-field anisotropy for these stable pat-

\section{June 2001}

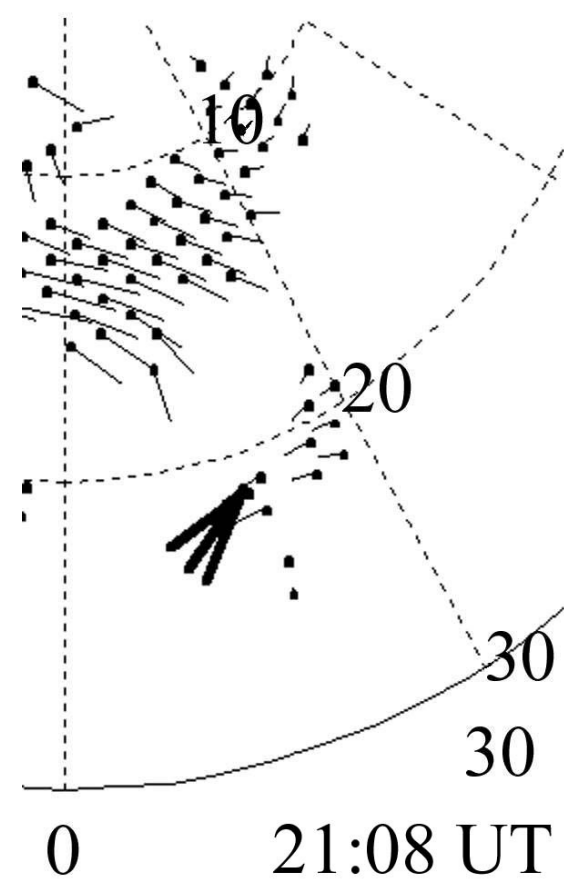

Fig. 9. SuperDARN convection map (thin lines originating from dots) for 5 June 2001 at 21:08 UT and the orientation of the cross-field anisotropy (thick lines) according to measurements at Futrikelv, Troms $\varnothing$ and Seljelvnes at 21:08 UT. The coordinates are geographic longitude and geographic co-latitude.

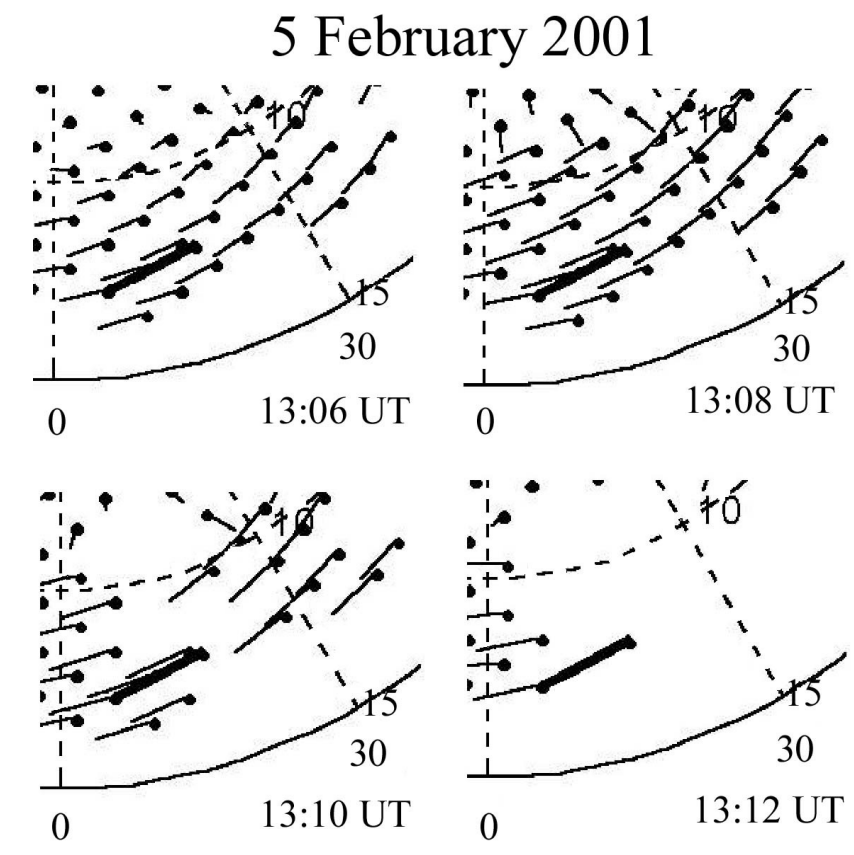

Fig. 10. The same as in Fig. 8 but for SuperDARN observations on 5 February 2001, 13:06-13:12 UT and for measurement of the orientation of the cross-field anisotropy at 13:10 UT. 

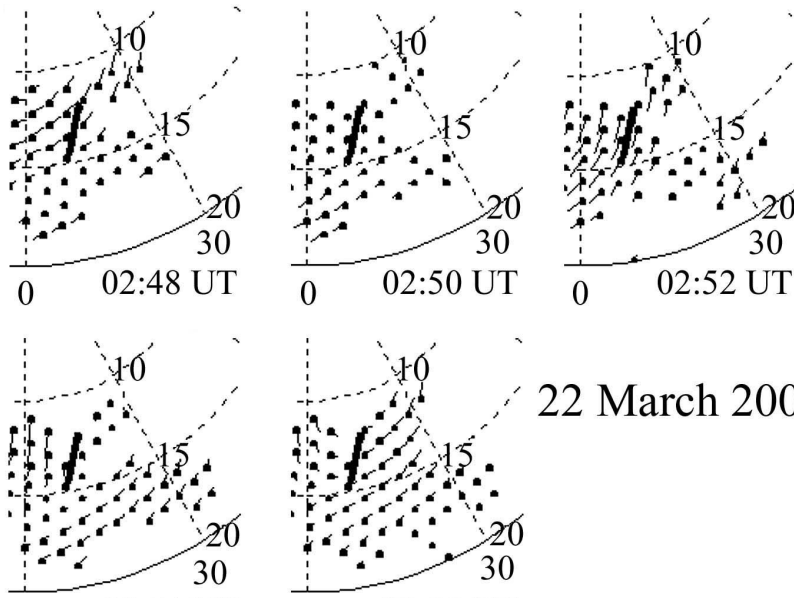

$02: 54 \mathrm{UT}$

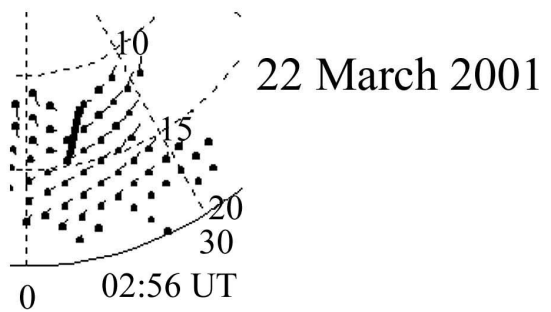

Fig. 11. The same as in Fig. 9 but for SuperDARN observations on 22 March 2001, 02:48-02:56 and the anisotropy orientation measurements at Barentsburg, Spitsbergen at 02:52 UT.

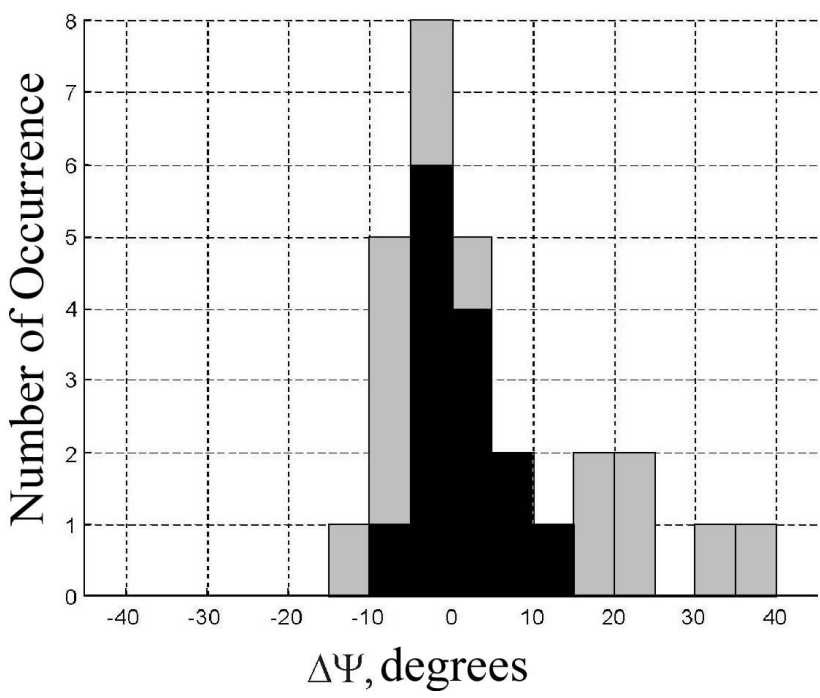

Fig. 12. Histogram distribution for the difference $\Delta \Psi$ between the orientation of the cross-field anisotropy and the direction of the plasma convection for observations at Barentsburg, Spitsbergen between September 2000 and November 2001. $5^{\circ}$ bins of the azimuth are used.

terns. This was not the case for non-stationary convection patterns.

Figure 11 compares SuperDARN and satellite data for an event of 22 March 2001, 02:48-02:56 UT for which the convection pattern was significantly changing. The scintillation measurements were performed at 02:52 UT. For the closest points, $\Psi=205^{\circ}\left( \pm 5^{\circ}\right)$ and $\Psi_{S D}=205^{\circ}(02: 52 \mathrm{UT})$, meaning that $\Delta \Psi=0^{\circ}$. For other frames, significant differences are obvious. This event demonstrates the importance of comparing radar and satellite data for as close as possible spatial areas and minimal difference in time. Clearly, utilization of the SuperDARN radars that can monitor the convection dynamics with a 2-min resolution is advantageous for the purposes of the present work; averaging over longer intervals can lead to smoothing out the short-lived local features in the convection map and more significant differences between the convection direction and the orientation of the cross-field anisotropy. On the other hand, this event lends additional support to the notion, first expressed by Tereshchenko et al. (2002) and further discussed in this study (Sect. 2.4), that significant differences in the orientation of the cross-field anisotropy at closely spaced points occur because of the local non uniformity in ionospheric convection flows.

Figure 12 presents statistics of the Barentsburg's comparisons for 28 events. The dark (grey) columns refer to those events for which the convection pattern was stable (nonstable); out of all events, in exactly half of them, the convection pattern was stable. One can see that the differences $\Delta \Psi$ were smaller for the stable convection patterns; for most of the events, $\Delta \Psi$ was within an interval of $-5^{\circ} \div 0^{\circ}$. For the non-stable convection events, the majority of the events also demonstrated relatively small differences, $\Delta \Psi$ was within $\pm 10^{\circ}$. For some events the differences were as large as $40^{\circ}$.

Our overall conclusion for the polar cap is that there is a reasonable agreement between the orientation of the crossfield anisotropy and the convection direction.

\section{Summary and conclusions}

In this study we further investigated the relationship between the orientation of the irregularity cross-field anisotropy and the plasma convection direction in the high-latitude ionosphere. The orientation of the cross-field anisotropy of irregularities was inferred from the amplitude scintillations of the satellite signals received on the ground. To achieve this, the latitudinal profile for the variance of the logarithm of the relative amplitude of the received signal was compared with the theoretically expected profiles, and the irregularity parameters corresponding to the best fit of the theory and experiment were obtained. The ionospheric plasma convection measurements were performed with the SuperDARN HF radars. We considered three different experiments, two in the auroral zone and one in the polar cap.

We demonstrated that the method of the irregularity parameters determination works well for observations not only in the auroral zone but also in the polar cap. To further improve the method, we investigated the effect of time averaging on the quality of model predictions; in previous studies, one instantaneous theoretical profile of signal fluctuations versus latitude has been used. We demonstrated that the time averaging has to be considered if one needs to estimate the elongation of the irregularities along and perpendicular to the geomagnetic field with better accuracy than from the time- independent model. Importantly, the time averaging does not affect the model estimates for the orientation of the cross-field anisotropy.

By comparing the satellite and SuperDARN data for three independent experiments we showed that the orientation of 
the irregularity cross-field anisotropy was fairly close, within $\pm 10^{\circ}$, to the direction of the plasma convection for the events with quasi-stationary convection pattern in the area of comparison. For the cases with quickly changing convection patterns, the agreement was satisfactory, with maximum differences of the order of $40^{\circ}$, if the comparison was performed for nearly the same moments. We can conclude that the ionospheric small-scale irregularities are elongated with the plasma convection direction in the plane perpendicular to the magnetic field. This conclusion is in line with expectations from the theory of the gradient-drift plasma instability in the high-latitude F region; it is predicted that as the instability progresses, plasma blobs experience stretching along the convection direction.

We also demonstrated that the multipoint satellite signal observations with the site separation of less than $100 \mathrm{~km}$ can be useful for studying the small-scale structures in the ionospheric plasma flows and thus provide additional information to the large-scale SuperDARN convection maps. The satellite scintillation data can successfully supplement the SuperDARN maps in those regions of the high-latitude ionosphere where the HF echoes are not detected. Finally, the multipoint satellite data may give information about the spatial uniformity of flows and their temporal stability.

Acknowledgements. The authors are grateful to J. A. Gorin for his help with SuperDARN data processing and to B. Z. Khudukon, Y. A. Mel'nichenko and V. M. Sukhorukov for their help in scintillation measurements. We thank all national funding agencies and individuals who make the SuperDARN convection maps available. This work was supported by the NSERC (Canada) grant to AVK and by the Russian Foundation of Fundamental Research grant No 03-05-64937.

Topical Editor M. Lester thanks two referees for their help in evaluating this paper.

\section{References}

Aarons, J.: Global morphology of ionospheric scintillations, Proc. of the IEEE, 70, 360-378, 1982.

Afraimovich, E. A., Lipko, Yu. V., and Vugmeister, B. O.: Parameters of small- and medium-scale irregularities in the high-latitude ionosphere as inferred from the Norilsk observations, Geomag. Aeron., 41, 120-127, 2001.

Eglitis, P., Robinson, T. R., Rietveld, M. T., Wright, D. M., and Bond, G. E.: The phase speed of artifical field-aligned irregularities observed by CUTLASS during HF modification of the auroral ionosphere, J. Geophys. Res., 103, 2253-2259, 1998.

Fremouw, E. J., Rino, C. L., Livingston, R. C., and Cousins, M. C.: A persistent subauroral scintillation enhancement observed in Alaska, Geophys. Res. Lett., 4, 539-542, 1977.

Gailit, T. A., Gusev, V. D., Ivanov, M. I., and Perekalina, E. O.: Differential-phase study of the anisotropy of ionospheric irregularities, Geomag. Aeron., 22, 622-625, 1982.

Gondarenko, N. A. and Guzdar, P. N.: Plasma patch structuring by the nonlinear evolution of the gradient drift instability in the high-latitude ionosphere, J. Geophys. Res., 109(A09301), doi:10.1029/2004JA010505, 2004.
Greenwald, R. A., Baker, K. B., Dudeney, J. R., Pinnock, M., Jones, T. B., Thomas, E. C., Villain, J.-P., Cerisier, J.-C., Senior, C., Hanuise, C., Hunsucker, R. D., Sofko, G., Koehler, J., Nielsen, E., Pellinen, R., Walker, A. D. M., Sato, N., and Yamagishi, H.: SuperDARN: A global view of the dynamics of high-latitude convection, Space Sci. Rev., 71, 763-796, 1995.

Gusev, V. D. and Ovchinnikova, N. P.: Model determination of the volumetric characteristics of ionospheric inhomogeneities, Geomagn. Aeron, 20, 434-437, 1980.

Keskinen, M. and Ossakow, S.: Theories of high-latitude ionospheric irregularities: a review, Radio Sci., 18, 1077-1091, 1983.

Martin, E. and Aarons, J.: F layer scintillations and the aurora, J. Geophys. Res., 82, 2717-2722, 1977.

Moorcroft, D. R. and Arima, K. S.: The shape of the F-region irregularities which produce satellite scintillations evidence for axial asymmetry, J. Atmos. Terr. Phys., 34, 437-450, 1972.

Rich, F. J. and Maynard, N. C.: Consequences of using simple analytical functions for the high-latitude convection electric field, J. Geophys. Res., 94, 3687-3701, 1989.

Rino, C. L., Livingston, R. C., and Matthews, S. J.: Evidence for sheet-like auroral ionospheric irregularities, Geophys. Res. Lett., 5, 1039-1042, 1978.

Rino, C. L. and Livingston, R. C.: On the analysis and interpretation of spaced-receiver measurements of transionospheric radio waves, Radio Sci., 17, 845-854, 1982.

Ruohoniemi, J. M., Greenwald, R. A., Baker, K. B., Villain, J. P., and McCready, M. A.: Drift motions of small-scale irregularities in the high-latitude F region: an experimental comparison with plasma drift motions, J. Geophys. Res., 92, 4553-4564, 1987.

Ruohoniemi, J. M. and Baker, K. B.: Large-scale imaging of highlatitude convection with SuperDARN HF radar observations, J. Geophys. Res., 103, 20 797-20 811, 1998.

Rytov, S. M., Kravtsov, Yu., A., and Tatarskii, V. I.: Introduction to statistical radio-physics. Part 2. Random Processes (in Russian). Nauka, Moscow, 1978.

Tereshchenko, E. D., Khudukon, B. Z., Kozlova, M. O., and Nygren, T.: Anisotropy of ionospheric irregularities determined from the amplitude of satellite signals at a single receiver, Ann. Geophys., 17, 508-518, 1999,

SRef-ID: 1432-0576/ag/1999-17-508.

Tereshchenko, E. D., Khudukon, B. Z., Kozlova, M. O., Evstafiev, O. V., Nygren, T., Rietveld, M. T., and Brekke, A.: Comparison of the orientation of small scale electron density irregularities and F region plasma flow direction, Ann. Geophys., 18, 918926, 2000a,

SRef-ID: 1432-0576/ag/2000-18-918.

Tereshchenko, E. D., Kozlova, M. O., Evstafiev, O. V., Khudukon, B. Z., Nygren, T., Rietveld, M., and Brekke, A.: Irregular structures of the F layer at high latitudes during ionospheric heating, Ann. Geophys., 18, 1197-1209, 2000b,

SRef-ID: 1432-0576/ag/2000-18-1197.

Tereshchenko, E. D., Romanova, N. Yu., and Kozlova, M. O.: Comparison of the cross-field anisotropy of small-scale irregularities with ionospheric convection model, Geomag. Aeron, 42, 432436, 2002.

Tereshchenko, E. D., Kozlova, M. O., Kunitsyn V. E., and Andreeva E. S.: Statistical tomography of subkilometer irregularities in the high-latitude ionosphere, Radio Sci., 39(RS1S35), doi:10.1029/2002RS002829, 2004.

Tsunoda, R. T.: High-latitude F region irregularities: A review and synthesis, Rev. Geophys., 26, 719-760, 1988. 